Address for Correspondence: Dr. Qing Wang,

Department of Gastroenterology, Qingdao Municipal Hospital,

Qingdao 266071, Shandong

Province, China.

E-mail: apple19790117@yahoo.cn

\begin{tabular}{|l|}
\hline Access this article online \\
\hline Website: \\
www.intern-med.com \\
\hline DOI: \\
10.1515/jtim-2013-0011 \\
\hline Quick Response Code: \\
\hline \\
\\
\end{tabular}

\title{
Marked elevation of CA19-9 in a patient with rheumatoid arthritis-associated pulmonary fibrosis
}

\author{
Wei-Wei Zhang, Qing Wang, Xiang-Jun Xie, Jun-Li Si \\ Department of Gastroenterology, Qingdao Municipal Hospital, Qingdao 266071, Shandong Province, China
}

\section{ABSTRACT}

We report a case of a 78-year-old male was admitted for 2 years of blood CA19-9 >1000 $\mathrm{kU} / \mathrm{L}$ found at physical examination. Abdominal computed tomography, barium meal and gastrointestinal endoscopy did not find any malignancy. Position emission tomography indicated interstitial pulmonary fibrosis (IPF) (severe) accompanied with reactive hyperplasia of mediastinal lymph node and bilateral pleural thickening. The patient also claimed to have rheumatoid arthritis (RA) for more than 40 years. Then final diagnosis was RA with IPF. The patient's blood CA19-9 was improved after 5-day treatment of hormone inhalation.

Key words: CA19-9, pulmonary fibrosis, rheumatoid arthritis

\section{INTRODUCTION}

CA19-9 is the monoclonal antibody of SW1116 human colon cancer antigen produced in mice. Here we report a marked elevation of CA19-9 in a case with rheumatoid arthritis-associated pulmonary fibrosis.

\section{CASE REPORT}

This was a case of a 78-year-old male patient who was admitted in July 2009 for "2 years of blood CA19-9 >1000 kU/L found at physical examination". During the last 2 years, the patient did not complain about abdominal pain or abdominal distension, no anorexia, no marasmus, no jaundice, no dark stools or hematemesis and the blood CA19-9 was measured to be $>1000 \mathrm{kU} / \mathrm{L}$ for many times. The patient visited the digestion department repeatedly for abdominal computed tomography (CT), whole digestive tract barium meal and gastroenterological endoscopy and no malignant diseases were found; then position emission tomography was performed, indicating interstitial pulmonary fibrosis (IPF) (severe) accompanied with reactive hyperplasia of mediastinal lymph node and bilateral pleural thickening. The patient's medical history was inquired and it was found that he had rheumatoid arthritis (RA) for more than 40 years and recurrent cough and shortness of breath for more than 2 years. Results of physical examination are listed below: Body temperature $36.7^{\circ} \mathrm{C}$, the sclera was not stained yellow, no swelling was found in systemic superficial lymph nodes, the distal ends of fingers were deformed, clubbed fingers and digits, coarse breathing sounds of both lungs, high inspiratory crepitus can be heard at both lower pulmonary fields and no abnormalities were found in the heart or abdomen. The routine blood, urine and stool examinations, hepatic and renal functions, blood fat, blood glucose, blood urine amylase, alpha-fetoprotein, antistreptococcus antibody, CA242, hemalexin C3 and C4 were all found to be normal, other results were: Immunoglobulin E 1190 $\mathrm{kU} / \mathrm{L}$, erythrocyte sedimentation rate 49 $\mathrm{mm} / \mathrm{h}$, blood C-reactive protein $33 \mathrm{mg} / \mathrm{L}$, rheumatoid factor $397 \mathrm{kU} / \mathrm{L}$, rheumatoid index antikeratin antibody weakly positive, anti-cyclic citrullinated peptide antibody $325 \mathrm{kU} / \mathrm{L}$, blood gas analysis $\mathrm{pH}$ 7.45, $\mathrm{PO}_{2}$ $78 \mathrm{mmHg}(1 \mathrm{mmHg}=0.133 \mathrm{kPa}), \mathrm{PCO}_{2}$ 
$38 \mathrm{mmHg}, \mathrm{SO}_{2} 96 \%$ and the pulmonary function test indicated moderate diffused and restrictive ventilatory functional disturbance. Diagnosis: RA, IPF. The patient's blood CA19-9 was improved (reduced to $901 \mathrm{kU} / \mathrm{L}$ ) after 5 -day treatment of hormone inhalation (budesonide atomization) to relieve breathlessness. The pancreatic and pulmonary CT images prior to treatment were shown in Figures 1 and 2, with further follow-up and observation being conducted.

\section{DISCUSSION}

CA19-9 is the monoclonal antibody of SW1116 human colon cancer antigen produced in mice, which is expressed as mucoprotein glycolipid in serum and expressed as GM1 ganglioside in tissues. Immunohistology has confirmed that trace amount of CA19-9 was present in normal human pancreas, bile duct, gall bladder, stomach, mammary gland and endometrium and it was one of the saccharide antigen tumor markers. CA19-9 was applied in the diagnosis of pancreatic cancer by Delvillano et al.

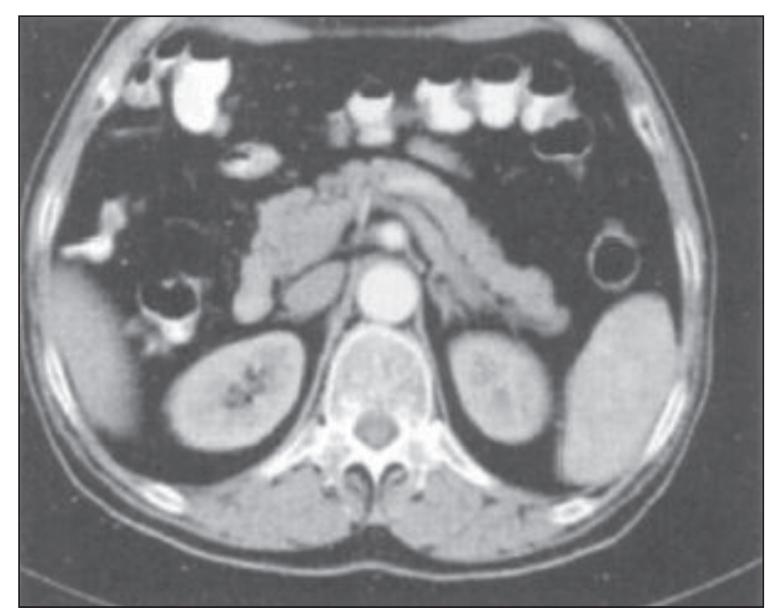

Figure 1: Pancreatic tomography indicated normal pancreatic morphology

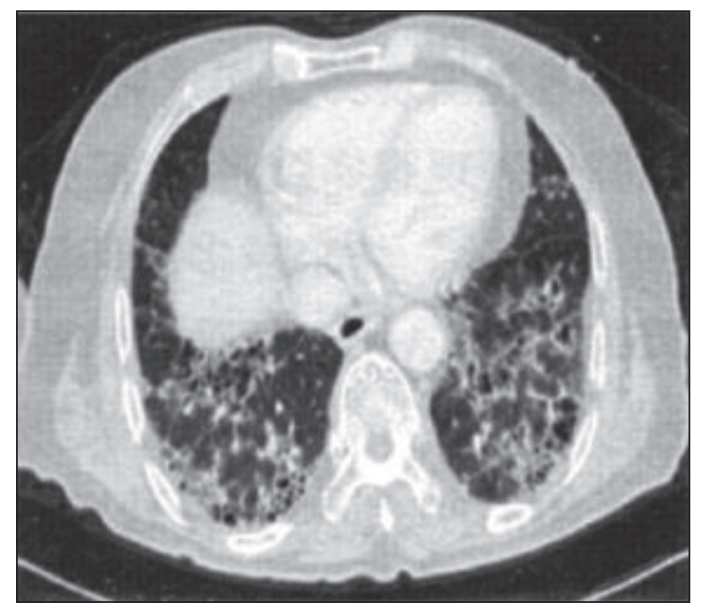

Figure 2: Pulmonary computed tomography indicated pulmonary interstitial fibrosis in 1983 and is currently widely applied in the differential diagnosis of malignant tumors in the digestive system. When CA19-9 is $>1000 \mathrm{kU} / \mathrm{L}$, the specificity of diagnosis of pancreatic cancer is higher than $95 \%$, which was also one of the reasons why this patient repeatedly visited the hospital and was monitored for digestive tumor. Since idiopathic pulmonary fibrosis with marked elevation of CA19-9 was reported in 6 Japanese patients by Fujita et al. ${ }^{[1]}$ in 1998 and one Chinese patient by Cao et al. ${ }^{[2]}$ in 2003, the public gained a further understanding of CA19-9, i.e., the elevation of CA19-9 was progressively increased with the aggravation of pulmonary fibrosis, indicating that it was also an index for pulmonary fibrosis progression and a marker of poor prognosis. This paper reports for the first time in China a case showing the relationship between marked elevation of CA19-9 and RA-associated IPF.

RA is a kind of systemic autoimmune disease with chronic symmetrical polyarthritis as a main clinical manifestation and it was found to be associated with interstitial lung disease in recent years. ${ }^{[3]}$ Some scholars believed that pulmonary parenchyma injuries as well as regeneration and repair caused by alveolus immunization and chronic inflammatory reaction were the basis of pathologic injury caused by RA associated IPF. The study conducted by Matsuoka and Cao suggested that injuries in physiologic barriers could be caused by inflammation in patients with pulmonary fibrosis and the CA19-9 elevation in serum and BALF were mainly sourced from injured bronchus and bronchiole epithelium and the squamous cells, pancake cells and cube epithelial cells metaplasted in the capsular space of the honeycomb structure and the compact fibrotic areas. This patient had a medical history of RA for many years, which was clearly diagnosed by vital signs and auxiliary examination, after hormone treatment the patient's symptom of breathlessness was improved and the serum CA19-9 was decreased.

With regards to the treatment of RA associated IPF, glucocorticoids was still the first choice, however, the therapeutic effects of IPF of different pathologic types and different phases were significantly different and the therapeutic effect was limited due to the patient's advanced age and the presence of certain side-effects with long-term administration of glucocorticoids.

\section{REFERENCES}

1. Fujita J, Obayashi Y, Yamadori I, Ohtsuki Y, Miyawaki H, Hojo S, et al. Marked elevation of CA19-9 in a patient with idiopathic pulmonary fibrosis: CA19-9 as a bad prognostic factor. Respirology 1998;3:211-4.

2. Cao SF, Cai SH, Liu CT. Idiopathic pulmonary fibrosis with marked elevation of CA19-9: A case report and literature review. J Clin Intern Med 2003;20:300-2. 
3. Kim EJ, Elicker BM, Maldonado F, Webb WR, Ryu JH, Van Uden JH, et al. Usual interstitial pneumonia in rheumatoid arthritis-associated interstitial lung disease. Eur Respir J 2010;35:1322-8.
How to cite this article: Zhang WW, Wang Q, Xie XJ, Si JL. Marked elevation of CA19-9 in a patient with rheumatoid arthritis-associated pulmonary fibrosis. J Transl Intern Med 2013; 1(1): 40-2.

Source of Support: Nil, Conflict of Interest: None declared 